\title{
Suppressing three-dimensional unsteady flows in vertical zone-melting by steady ampoule rotation
}

\author{
C.W. Lan*, M.C. Liang, C.H. Chian \\ Department Of Chemical Engineering, National Taiwan University, Taipei 10617, Taiwan \\ Received 18 November 1999; accepted 11 March 2000 \\ Communicated by D.T.J. Hurle
}

\begin{abstract}
Three-dimensional (3D) unsteady heat flow and molten zone induced by nonlinear bifurcations or imperfect growth conditions are typical in vertical zone-melting crystal growth or refining. Through flow modification, we find that the use of steady ampoule rotation is effective in suppressing these 3D characteristics. Even with only $10 \mathrm{RPM}$ ampoule rotation, numerical simulation indicates that the steady axisymmetric (or nearly) mode can be easily retained for 2-in GaAs under a typical growth condition, in which the $3 \mathrm{D}$ unsteady flows prevail for no rotation. A model gallium zone is also examined, and its 3D bifurcations are significantly suppressed by rotation as well. However, if the rotation is not sufficient to suppress the unsteadiness, baroclinic waves may appear. The effects of ampoule rotation for imperfect growth conditions, including asymmetric heating and ampoule tilting, are further illustrated. The ampoule rotation is also found useful. (C) 2000 Elsevier Science B.V. All rights reserved.
\end{abstract}

PACS: 44.25. + f; 47.27.Te; 81.10.Fq; 02.60.Cb; 02.70.Fj

Keywords: 3D flows; Rotation; Zone-melting; Interfaces; Asymmetric heating; Ampoule tilting; Flow control

\section{Introduction}

The vertical zone melting (VZM) is an important process for both crystal growth and purification [1-4]. However, because of the radial zone heating, the induced thermal convection is strong. As a result, not only can the growth interface be significantly affected $[5,6]$, but also three-dimensional (3D) nonlinear bifurcations, such as symmetry

\footnotetext{
* Corresponding author. Tel.: +886-2-2363-3917; fax: + 886-2-2363-3917.

E-mail address: lan@ruby.che.ntu.edu.tw (C.W. Lan).
}

breaking and unsteadiness, may prevail [7-9]. The $3 \mathrm{D}$ heat flows as well as the resulted growth interface may have significant influences, usually detrimental, on the crystal quality. An imperfect growth condition, such as ampoule tilting or asymmetric heating, also induces the 3D flows and the molten zone. How to suppress and control these 3D characteristics is a great interest for crystal growers.

By studying an ideal gallium melt zone, both numerically and experimentally, Neumann [7] and Baumgartl et al. [8] found that with increasing Rayleigh number $\left(\mathrm{Ra}_{\mathrm{w}}\right)$, a steady axisymmetric mode bifurcated to a 3D toroidal mode first at $\mathrm{Ra}_{\mathrm{w}} \approx 3 \times 10^{4}$ and then to a time-dependent mode 
at a higher $\operatorname{Ra}_{\mathrm{w}}$ (about $4 \times 10^{4}$ ) for a parabolic thermal profile at the melt surface. A steady oneroll (m1) mode was found for $8 \times 10^{4}<$ $\mathrm{Ra}_{\mathrm{w}}<1.85 \times 10^{5}$. Beyond $\mathrm{Ra}_{\mathrm{w}}=1.85 \times 10^{5}$, the $\mathrm{m} 1$ flow became time dependent. The growth of GaAs single crystals was also carried out at $\mathrm{Ra}_{\mathrm{w}} \approx 4 \times 10^{5}$, and $3 \mathrm{D}$ striation patterns were found [8]. In a recent numerical study by Lan and Liang [9], they found that the symmetry breaking was subcritical for GaAs, and the subcriticality might be caused by the deformable interfaces. Although their work mainly focused on the steady symmetry breaking, 3D unsteady $\mathrm{m} 1$ flows were also found for $\mathrm{Ra}_{\mathrm{w}}>2.23 \times 10^{5}$, which was consistent with the previous growth experiment. To avoid the unsteadiness, a lower-superheating and shorter zone was needed. However, the use of a shorter zone or lower thermal gradients is not quite feasible in practice due to the difficulty in the process control.

Besides the inherent symmetry breaking, in reality, the 3D characteristics can also be induced by imperfect growth conditions, such as asymmetric heating or ampoule tilting. By imposing an asymmetric thermal boundary condition, Baumgartl et al. [8] also found that only the asymmetric mode could exist, and the rotational $\mathrm{m} 0$ flow disappeared. Significant 3D flow and dopant segregations for vertical Bridgman (VB) crystal growth due to the asymmetric environments are also typical, as illustrated by Xiao et al. [10] and Liang and Lan [11]. For a better process control, it may be of importance to suppress the 3D flows. Using a magnetic field to suppress the 3D flows has been found feasible for both VZM and VB growth (see e.g., Refs. $[12,13])$. With a high enough magnetic field strength, Baumgartl et al. [12] have also illustrated the transition from an unsteady $\mathrm{m} 1$ mode to a steady m0 mode for a model gallium system at $\mathrm{Ra}_{\mathrm{w}}=2.5 \times 10^{5}$. Even for horizontal zone-melting growth, Muller [14] also illustrated the usefulness of a transversal magnetic field to damp the unsteady flows. Nevertheless, the use of magnetic fields is still too expensive in applications.

Recently, Lan and Chian [15] studied the effects of 5-25 RPM ampoule rotation on the 2D heat flows and the zone shape during VZM growth of a 2-in GaAs crystal. They found that the steady ampoule rotation was effective in modifying the local flow near the center of the growth interface. As a result, a convex interface could be obtained easily, even with only 5 RPM rotation. Clearly, the role of the low-speed ampoule rotation is not to damp the bulk flow. Instead, it seems to be more effective in modifying the local flow structure. Therefore, it may be of interest to try that for suppressing the 3D characteristics. In theory [16], it is possible to suppress the buoyancy effect by the Coriolis force. However, a too-high rotation speed causes mechanical vibrations that may be detrimental to the growth as well. Also, if the final interfaces are not axisymmetric, the rotation may cause significant regrowth and remelting, which could make the growth even worse. Furthermore, the baroclinic instability due to the coupling of the Coriolis and buoyancy forces may be induced. Therefore, care must be taken for using ampoule rotation in practice.

In this study, we will examine the effects of lowspeed steady ampoule rotation on the 3D nature in VZM crystal growth. Since such an idea has not yet been tested before, to provide more convincing results, we will first use a commercial code Fluent [17] to examine the effects of rotation for the simplified model zone used by Baumgartl et al. [8]. Detailed numerical simulation for GaAs growth using a more realistic model is then carried out. Besides the inherent bifurcations, the influence of rotation on the heat flow under imperfect growth conditions is then illustrated. In the next section, the mathematical model and its numerical solution are described briefly. Section 3 is devoted to results and discussion, followed by conclusions and comments in Section 4.

\section{Model description and numerical solution}

A generic VZM crystal growth system is illustrated in Fig. 1a. Since axisymmetry is no longer assumed here, the system is described by a fixed Cartesian coordinate $(x, y, z)$. The zone heater is described by an effective heating profile $T_{\mathrm{a}}(x$, $y, z, t)$, which is kept stationary in this study. For pseudo-steady-state calculations, the ampoule pulling speed $U_{\text {amp }}$ is set to be the steady melting and 


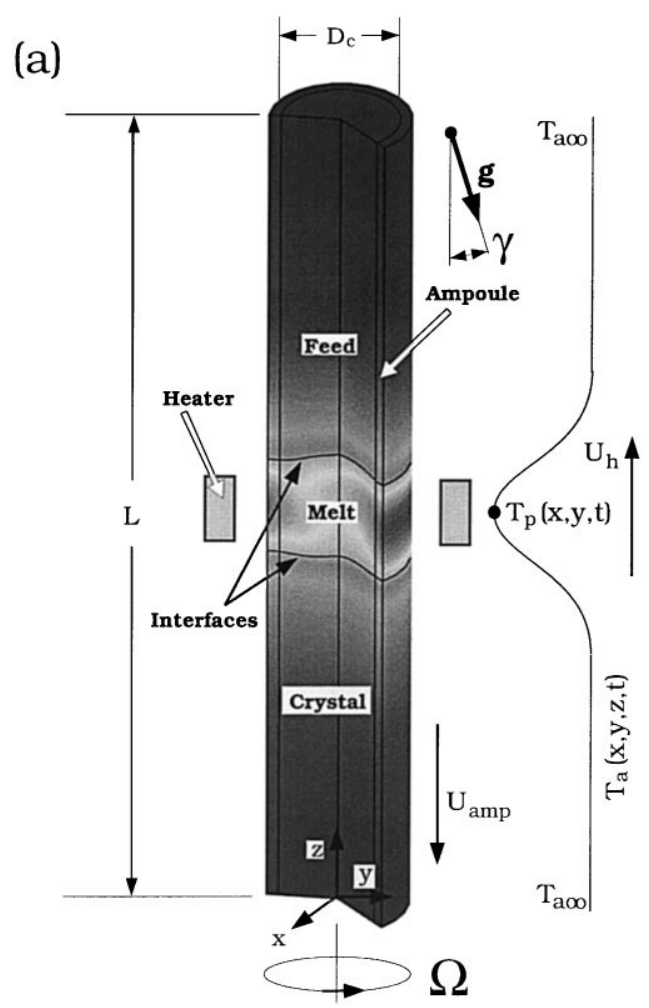

(b)

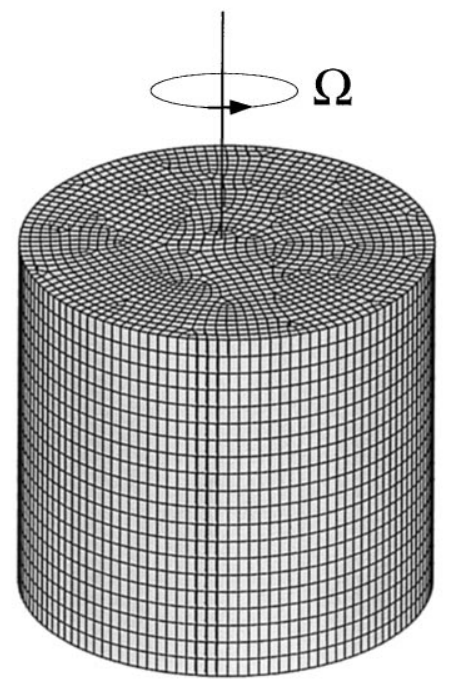

Parabolic thermal profile at melt surface

Fig. 1. (a) Schematic of vertical zone-melting (VZM) crystal growth; (b) a simplified model and mesh for gallium melt zone.

growth rates. Such an assumption is reasonable and can reduce computation load significantly [18]. The ampoule rotation rate $\Omega$ is fixed here; variable speeds are allowed. The flow and temperature fields as well as the melt/feed $\left(h_{\mathrm{f}}(x, y, z, t)\right)$ and melt/crystal $\left(h_{\mathrm{c}}(x, y, z, t)\right)$ interfaces are also represented by the Cartesian coordinate.

The dimensionless variables are defined by scaling length with crystal diameter $D_{\mathrm{c}}$, time $t$ with $D_{\mathrm{c}}^{2} / \alpha_{\mathrm{m}}$, velocity with $\alpha_{\mathrm{m}} / D_{\mathrm{c}}$, pressure with $\rho_{\mathrm{m}} \alpha_{\mathrm{m}}^{2} / D_{\mathrm{c}}^{2}$, and temperature $T$ with melting point $T_{\mathrm{m}}$, where $\alpha_{\mathrm{m}}$ is the thermal diffusivity and $\rho_{\mathrm{m}}$ the melt density. The time-dependent governing equations describing the convection and heat transport in the melt $(\mathrm{m})$ are as follows:

$\nabla \cdot v=0$,

$\partial \boldsymbol{v} / \partial \tau+\boldsymbol{v} \cdot \nabla \boldsymbol{v}=-\nabla P+\operatorname{Pr} \nabla^{2} \boldsymbol{v}$

$$
-\operatorname{Pr} \operatorname{Ra}_{\mathrm{T}}(\theta-1) \boldsymbol{e}_{\mathrm{g}},
$$

$\partial \theta / \partial \tau+\boldsymbol{v} \cdot \nabla \theta=\nabla^{2} \theta$ where $\tau, \boldsymbol{v}, P$, and $\theta$ are the dimensionless time, velocity, pressure, and temperature, respectively. $\mathrm{Pr}$ is the Prandtl number and $\boldsymbol{e}_{\mathrm{g}}$ the unit vector in the gravity direction. For a tilted ampoule, the growth direction is deviated from $\boldsymbol{e}_{\mathrm{g}}$ by an angle $\gamma$, as shown in Fig. 1a. The associated dimensionless number $\mathrm{Ra}_{\mathrm{T}}$ in the source term of the momentum equation is the thermal Rayleigh number, defined as follows:

$$
\mathrm{Ra}_{\mathrm{T}} \equiv \frac{g \beta_{\mathrm{T}} T_{\mathrm{m}} D_{\mathrm{c}}^{3}}{\alpha_{\mathrm{m}} v_{\mathrm{m}}},
$$

where $g$ is the gravitational acceleration, $\beta_{\mathrm{T}}$ the thermal expansion coefficients, and $v_{\mathrm{m}}$ the kinematic viscosity. This definition is somewhat different from the one used by Baumgartl et al. [8]. Their Rayleigh number was defined as follows:

$\mathrm{Ra}_{\mathrm{w}} \equiv \frac{2 g \beta_{\mathrm{T}} \Delta T D_{\mathrm{c}}^{3}}{\alpha_{\mathrm{m}} v_{\mathrm{m}}}$, 
where $\Delta T$ is the maximum temperature difference in the melt.

In the crystal (c), the feed (f), and the ampoule (a), only heat transfer needs to be considered:

$$
\begin{aligned}
& \partial \theta / \partial \tau+\left(-v_{\text {amp }} \boldsymbol{e}_{z}+r \Omega^{*} \boldsymbol{e}_{\phi}\right) \cdot \nabla \theta=\tilde{\kappa}_{i} \nabla^{2} \theta \\
& \quad(i=\mathrm{c}, \mathrm{f}, \mathrm{a}),
\end{aligned}
$$

where $\tilde{\kappa}_{i} \equiv \alpha_{i} / \alpha_{\mathrm{m}}$ is the dimensionless thermal diffusivity of the feed ( $i=\mathrm{f})$, crystal $(i=\mathrm{c})$ or ampoule $(i=\mathrm{a}) ; \alpha_{i}$ is the thermal diffusivity $(i=\mathrm{f}, \mathrm{c}$, or $a)$. Also, $v_{\text {amp }}$ is the dimensionless ampoule pulling speed, $\Omega^{*}$ the dimensionless rotation speed, and $\boldsymbol{e}_{z}$ and $\boldsymbol{e}_{\phi}$ are the unit vectors in the axial and angular directions, respectively.

The no-slip condition is used for the melt velocity on solid boundaries,

$\boldsymbol{v}=\gamma_{\mathrm{c}}\left(-v_{\mathrm{amp}} \boldsymbol{e}_{z}+r \Omega^{*} \boldsymbol{e}_{\phi}\right)$,

where $\gamma_{\mathrm{c}} \equiv \rho_{\mathrm{c}} / \rho_{\mathrm{m}} ; \gamma_{\mathrm{c}}=1$ is used here.

The thermal boundary conditions at the growth and feeding fronts are set by the heat flux balances

$$
\begin{gathered}
\left.Q\right|_{\mathrm{m}}-\left.Q\right|_{\mathrm{c}}+\gamma_{\mathrm{c}}\left[\left(v_{\mathrm{amp}}+\mathrm{d} h_{\mathrm{c}} / \mathrm{d} \tau\right) \boldsymbol{e}_{z}\right. \\
\left.-r \Omega^{*} \boldsymbol{e}_{\phi}\right] \mathrm{St} \cdot \boldsymbol{n}=0, \\
\left.Q\right|_{\mathrm{m}}-\left.Q\right|_{\mathrm{f}}+\gamma_{\mathrm{c}}\left[\left(v_{\mathrm{amp}}+\mathrm{d} h_{\mathrm{f}} / \mathrm{d} \tau\right) \boldsymbol{e}_{z}\right. \\
\left.-r \Omega^{*} \boldsymbol{e}_{\phi}\right] \mathrm{St} \cdot \boldsymbol{n}=0,
\end{gathered}
$$

where $\boldsymbol{n}$ is the unit normal vector at the feed or growth interface pointing to the melt. $\left.Q\right|_{\mathrm{m}},\left.Q\right|_{\mathrm{c}}$, and $\left.Q\right|_{\mathrm{f}}$ are the dimensionless total heat fluxes at the melt, the crystal, and the feeding sides, respectively. The Stefan number $\mathrm{St} \equiv \Delta H / C p_{\mathrm{m}} T_{\mathrm{m}}$ scales the heat of fusion $(\Delta H)$ released during solidification to the sensible heat in the melt; $C p_{\mathrm{m}}$ is the specific heat of the melt. The component for $\left(r \Omega^{*} \boldsymbol{e}_{\phi} \cdot \boldsymbol{n}\right)$ is particularly important for 3D interfaces during rotation. Clearly, the melting $\left(\boldsymbol{e}_{\phi} \cdot \boldsymbol{n}>0\right)$ and growth $\left(\boldsymbol{e}_{\phi} \cdot \boldsymbol{n}<0\right)$ rates may be larger than the ampoule pulling rate if $\left(\boldsymbol{e}_{\phi} \cdot \boldsymbol{n}\right)$ is not trivial. Furthermore, this term also increases the interface distortion along the rotational direction, which can further reduce the asymmetry.

The heat exchange between the ampoule and the furnace is by both radiation and convection according to the energy balance along the ampoule surface,

$$
-\left.\boldsymbol{n} \cdot \kappa_{\mathrm{a}} \nabla \theta\right|_{\mathrm{a}}=\operatorname{Bi}\left(\theta-\theta_{\mathrm{a}}\right)+\operatorname{Rad}\left(\theta^{4}-\theta_{\mathrm{a}}^{4}\right),
$$

where $\boldsymbol{n}$ is the unit normal vector on the ampoule surface pointing outwards, $\mathrm{Bi} \equiv h D_{\mathrm{c}} / k_{\mathrm{m}}$ the Biot number, and $\operatorname{Rad} \equiv \sigma \varepsilon_{\mathrm{a}} T_{\mathrm{m}}^{3} D_{\mathrm{c}} / k_{\mathrm{m}}$ the radiation number; $h$ is the heat transfer coefficient, $k_{\mathrm{m}}$ the melt thermal conductivity, $\sigma$ is the Stefan Boltzmann constant, and $\varepsilon_{\mathrm{a}}$ is the surface emissivity of the ampoule. For simplicity, the effective heater temperature $\theta_{\mathrm{a}}$ is assumed to be a Gaussian distribution

$$
\begin{aligned}
\theta_{\mathrm{a}}(x, y, z, t)= & \theta_{\mathrm{a} \infty}+\left(\theta_{\mathrm{p}}-\theta_{\mathrm{a} \infty}\right) \\
& \times \exp \left(-\left[\left(z-z_{\mathrm{p}}\right) / a\right]^{2}\right),
\end{aligned}
$$

where $\theta_{\mathrm{a} \infty}$ and $\theta_{\mathrm{p}}$ are the dimensionless ambient background and peak temperatures, respectively, and $a$ is related to the width of distribution. For the case with asymmetric heating, angular variation of $\theta_{\mathrm{p}}$ is further considered

$\theta_{\mathrm{p}}(x, y)=\left.\theta_{\mathrm{p}}\right|_{\phi=0}-\Delta \theta_{\mathrm{p}} \cos \phi$,

where $\Delta \theta_{\mathrm{p}}$ is the deviation from the average peak temperature.

The above governing equations and their associated boundary conditions can only be solved numerically. We have developed efficient finitevolume method schemes using the primitive variable formulation [19] and multigrid acceleration [20] for the free or moving boundary problem. The second-order central difference scheme is adopted for both convective and diffusion terms using the deferred correction. For time-dependent calculations, an implicit Euler scheme [21] is used for time integration. Detailed numerical schemes and benchmark comparison with Fluent and the 2D stream function/vorticity code [18] can be found in Ref. [9].

\section{Results and discussion}

Before presenting the results for crystal growth, we have performed a series of numerical simulations using Fluent for the gallium melt zone studied by Baumgartl et al. [8]. For the ideal zone, a parabolic temperature profile is specified at the melt surface, while the top and bottom boundaries are set at a fixed temperature. Clear symmetry breaking and unsteadiness have also been reported $[7,8]$. 
Therefore, this model serves as an excellent candidate for examining the effects of rotation on the flow bifurcations as well as for benchmarking. The unstructured mesh used for this case (aspect ratio of one) is shown in Fig. 1b. There are 28440 control volumes and this leads to 142200 unknowns; finer meshes (up to 77370) have also been used, but the result is not changed much; the difference on the maximum center temperature is within $1 \%$. Although $\mathrm{Ra}_{\mathrm{T}}$, Ta and $\mathrm{Pr}$ are the only dimensionless numbers for describing this system, we need to give dimensional values for using Fluent; $\mathrm{Ta} \equiv$ $\Omega^{2} D_{\mathrm{c}}^{4} / v_{\mathrm{m}}$. Therefore, $D_{\mathrm{c}}=2 \mathrm{~cm}$ and $\Delta T=265.7^{\circ} \mathrm{C}$ are used, and the Rayleigh number $\mathrm{Ra}_{\mathrm{w}}$ is adjusted through the gravitational acceleration. In addition, $\operatorname{Pr}=0.02$ is also used. Since the Boussinesq ap- proximation and constant physical properties are used, the calculated results will depend on the dimensionless numbers $\left(\mathrm{Ra}_{\mathrm{w}}, \mathrm{Ta}, \mathrm{Pr}\right)$ and the zone aspect ratio only. To start the calculation, the solution for $g=\Omega=0\left(\right.$ or $\left.\mathrm{Ra}_{\mathrm{w}}=\mathrm{Ta}=0\right)$ is used as the initial condition, i.e., conduction only. Time integration is then started by specifying the gravity acceleration and the rotation speed. Second-order schemes are used both in the space and time. In addition, no relaxation is used for temperature (relaxation factor being one); the relaxation in temperature degrades the convergence rate dramatically.

At $\mathrm{Ra}_{\mathrm{w}}=7.32 \times 10^{5}$, which is way beyond the onset point of the unsteady $\mathrm{m} 1$ mode $\left(\mathrm{Ra}_{\mathrm{w}} \approx 2 \times 10^{5}\right)$, we obtain very chaotic flows. As shown by the solid line in Fig. 2, without rotation,

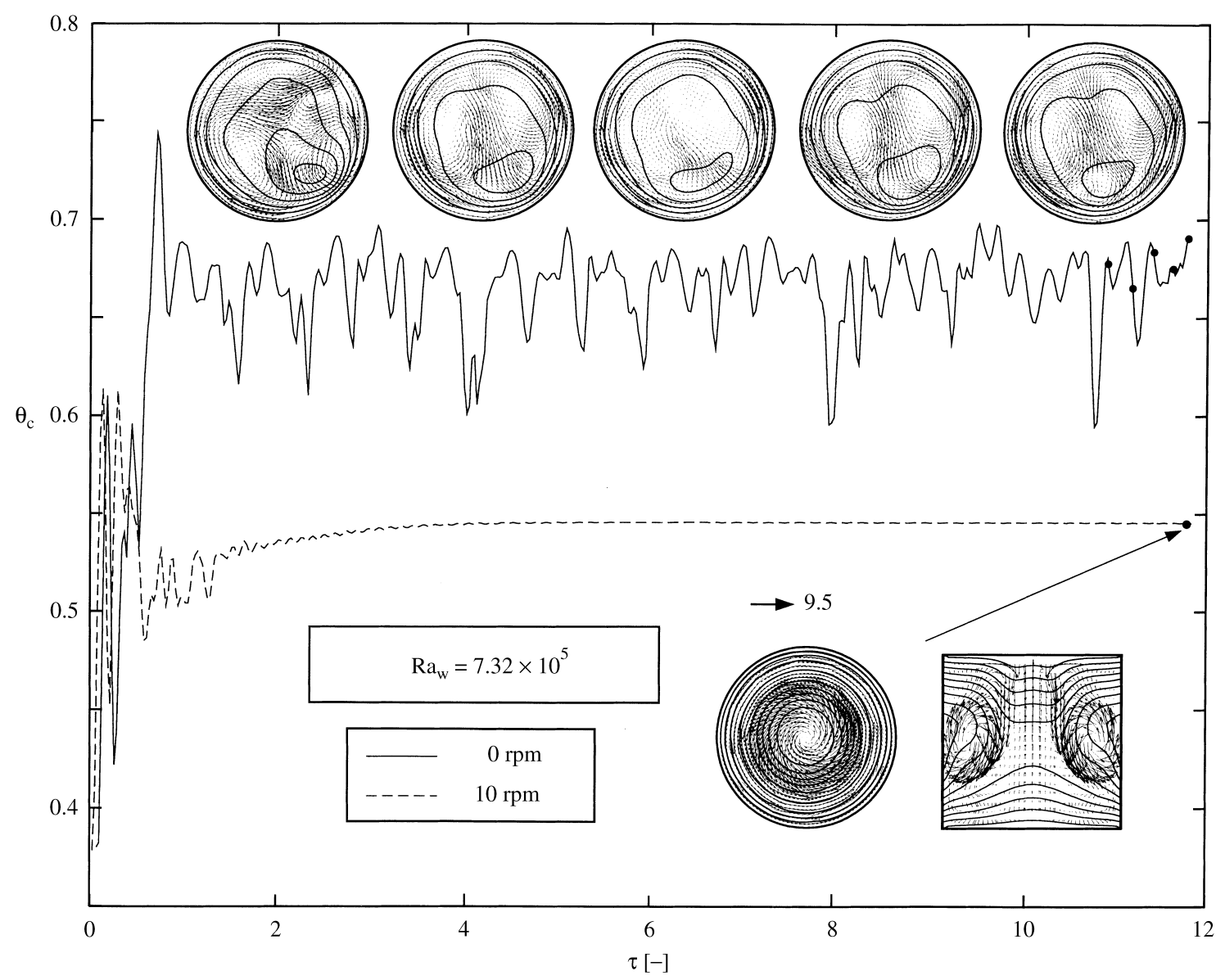

Fig. 2. Temperature responses at the zone center for 0 and $10 \mathrm{RPM}(\mathrm{Ta}=2056.49)$; some flow patterns and isotherms at the mid-plane are shown; $\mathrm{Ra}_{\mathrm{w}}=7.32 \times 10^{5}$. 
the temperature oscillation is very irregular, where $\theta_{\mathrm{c}}$ is the redefined dimensionless temperature at the zone center; $\theta_{\mathrm{c}}=\left(T_{\mathrm{c}}-T_{\mathrm{m}}\right) /\left(T_{\max }-T_{\mathrm{m}}\right)$. Some flow structures (vector fields) and mid-plane isotherms near the end of calculation are shown in the upper part of the figure. As shown, the calculated flow structures without rotation are all $\mathrm{m} 1$. As reported before $[7,8]$, the flows are not rotational. One can examine the flow structure, the flow is moving back and forth only with time. With $10 \mathrm{RPM}$ rotation (or $\mathrm{Ta}=2056.49$ ), as shown by the dashed line in Fig. 2, the temperature finally reaches to a steady state and the flow mode is $\mathrm{m} 0$. Here, the "steady state" means that its maximum dimensionless temperature variation is within $1 \times 10^{-3}$. The axisymmetric mode can be better examined by the mid-plane isotherms. From the side view, there is an upward flow at the zone center and this is due to ampoule rotation, as illustrated by Lan and Chian [15]. Furthermore, from the isotherms near the lower interface, during crystal growth the interface shape will be convex as well. From Fig. 2, clearly, the steady ampoule rotation indeed significantly suppresses the symmetry breaking and unsteadiness. The convex isotherms near the growth boundary are also beneficial.

Of course, the 10 RPM rotation is not always sufficient to suppress the $3 \mathrm{D}$ unsteady flows if $\mathrm{Ra}_{\mathrm{w}}$ is further increased. In fact, $\mathrm{Ra}_{\mathrm{w}}=7.32 \times 10^{5}$ is right at the margin already. Fig. 3 shows the result at $\mathrm{Ra}_{\mathrm{T}}=9.15 \times 10^{5}$. As shown, with $10 \mathrm{RPM}$ rotation, the melt temperature is still oscillating, but it becomes much more regular than that in Fig. 2; the oscillation period is about 0.4208 (or $16 \mathrm{~s}$ ). More interestingly, if we further examine the one-period flow and thermal fields, as shown in the same

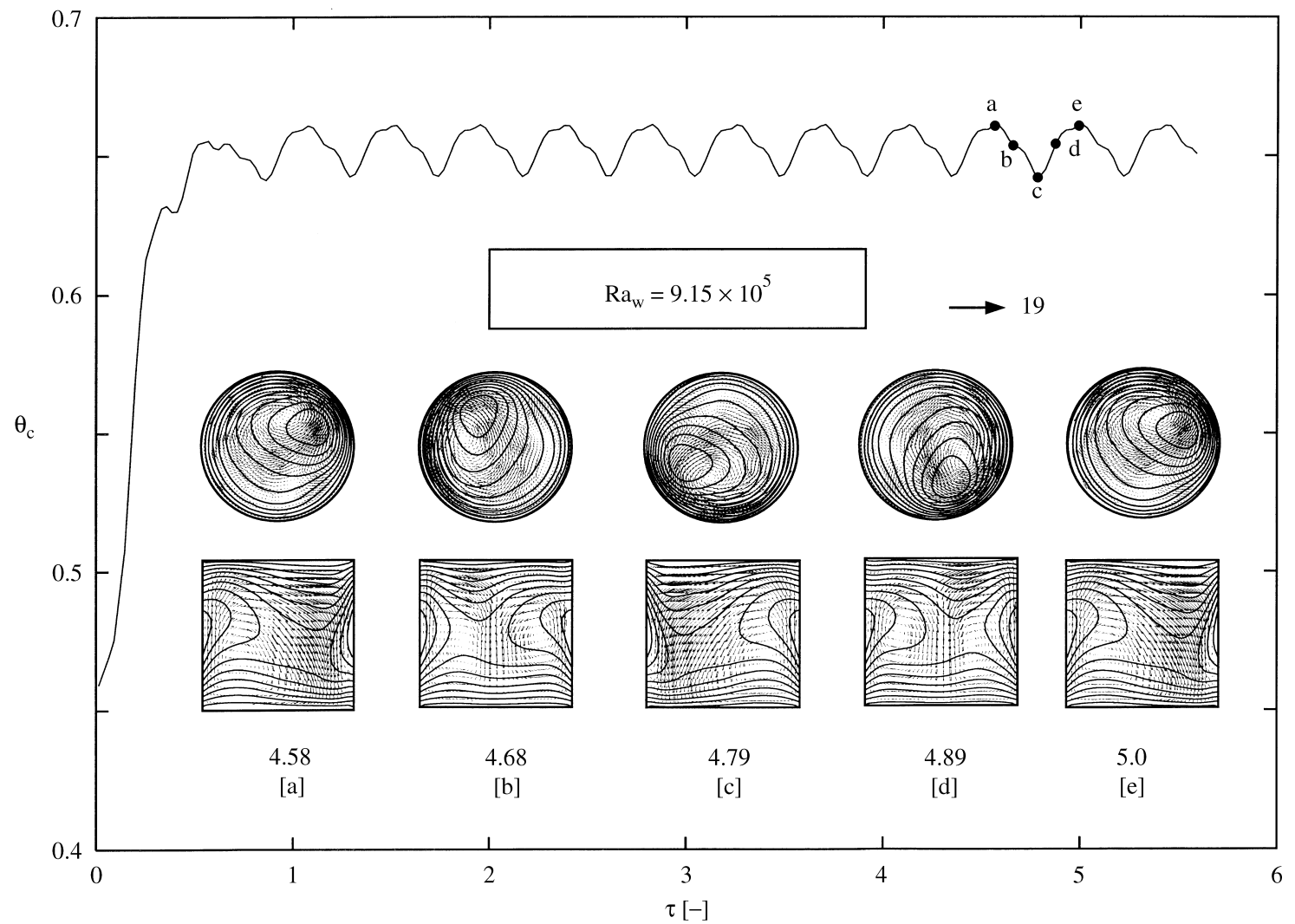

Fig. 3. Temperature response at the zone center for $\mathrm{Ra}_{\mathrm{w}}=9.15 \times 10^{5}$ with $10 \mathrm{RPM}$ rotation $(\mathrm{Ta}=2056.49)$. Some flow patterns and isotherms at the mid-plane from a to e are also shown. 
figure, the time-dependent flow is rotational, i.e., with an angular wave. This is the so-called baroclinic wave, which is caused by the interaction between the buoyancy and Coriolis forces [22,23]. This angular wave travels at a lower speed than the applied rotation rate; 10 RPM rotation takes $6 \mathrm{~s}$ (or $\tau=0.1578$ ) for one revolution. Again, without rotation, the unsteady flows are similar to that in Fig. 2 (upper field plots). No angular wave is found as well. The temperature oscillation is also quite chaotic.

From this simplified model, it is clear that, though quite unexpected, the 10 RPM rotation may suppress the $3 \mathrm{D}$ bifurcations, both symmetry breaking and unsteadiness. In fact, the magnetic filed strength used by Baumgartl et al. [12] for suppressing the $3 \mathrm{D}$ unsteady flow was also very low; $\mathrm{Ha} \approx 30$ or $B \approx 0.05 \mathrm{~T}$, where $\mathrm{Ha}$ is the Hartmann number and $B$ the magnetic field strength. Nevertheless, as compared with magnetic damping, steady ampoule rotation is much more feasible in practice and simpler. To further illustrate the idea, we also conduct calculations using a more realistic model [9] for GaAs, where the melting and growth interfaces are considered. The physical properties and some input parameters are listed in Table 1 for references [9,24-27]. Detailed bifurcations for both high and low superheatings using the gravity or peak heater temperature as the parameter can be found in Ref. [9]. For the high-superheating case at $T_{\mathrm{p}}=1600^{\circ} \mathrm{C}$, the bifurcation diagram from Ref. [9] shows a Hopf bifurcation at $\mathrm{Ra}_{\mathrm{w}} \approx 2.23 \times 10^{5}$ or $0.26 \mathrm{~g}$, as shown in Fig. $4 \mathrm{a} ; \Delta h$ is the zone-length deviation and it is zero for an axisymmetric zone. At normal gravity $\left(\mathrm{Ra}_{\mathrm{w}} \approx 7.36 \times 10^{5}\right)$, the oscillating flow also have significant effects on the zone length (inside Fig. 4a), as well as on the periodic growth and melting. As shown by the zone length oscillation, the long period is about $88 \mathrm{~s}$. This value increases with decreasing gravity. Near the onset point, at $g=0.3$, the oscillation period is about $450 \mathrm{~s}$.

One period of oscillation for the isotherms is shown in Fig. 4b, where both the mid-plane isotherms and isotherm surfaces are shown. A typical flow structure is illustrated in Fig. 5a for reference. As shown in Fig. 5a, there are two major and one small flow cells in the molten zone. During oscilla-
Table 1

Physical properties and some input parameters [9,24-27]
GaAs

$\rho_{\mathrm{c}}=\rho_{\mathrm{f}}=5.7 \mathrm{~g} \mathrm{~cm}^{-3}$

$\rho_{\mathrm{m}}=5.7 \mathrm{~g} \mathrm{~cm}^{-3}$

$T_{\mathrm{m}}=1238^{\circ} \mathrm{C}$

$\Delta H=726 \mathrm{~J} \mathrm{~g}^{-1}$

$h=1.6 \times 10^{-2} \mathrm{~W} \mathrm{~cm}^{-2}{ }^{\circ} \mathrm{C}^{-1}$

$k_{\mathrm{c}}=k_{\mathrm{f}}=0.07 \mathrm{~W} \mathrm{~cm}^{-1}{ }^{\circ} \mathrm{C}^{-1}$

$k_{\mathrm{m}}=0.14 \mathrm{~W} \mathrm{~cm}^{-1}{ }^{\circ} \mathrm{C}^{-1}$

$C p_{\mathrm{c}}=C p_{\mathrm{f}}=C p_{\mathrm{m}}=0.42 \mathrm{~J} \mathrm{~g}^{-1}{ }^{\circ} \mathrm{C}^{-1}$

$\beta_{\mathrm{T}}=1.16 \times 10^{-4} \mathrm{~K}^{-1}$

Quartz

$\rho_{\mathrm{a}}=2.2 \mathrm{~g} \mathrm{~cm}^{-3}$

$k_{\mathrm{a}}=0.035 \mathrm{~W} \mathrm{~cm}^{-1}{ }^{\circ} \mathrm{C}^{-1}$

$C p_{\mathrm{a}}=0.188 \mathrm{~J} \mathrm{~g} \mathrm{~g}^{-1}{ }^{\circ} \mathrm{C}^{-1}$

$\varepsilon_{\mathrm{a}}=0.8$

Other input parameters

$L=15 \mathrm{~cm}$

$D_{\mathrm{c}}=2 \mathrm{~cm}$

$D_{\mathrm{a}}=2.4 \mathrm{~cm}$

$T_{\mathrm{p}}=1600^{\circ} \mathrm{C}$ (for high superheating);

$1500^{\circ} \mathrm{C}$ (for low superheating)

$T_{\mathrm{a} \infty}=300^{\circ} \mathrm{C}$

$2.0 \mathrm{~cm}$ (for low superheating)

$a_{\mathrm{f}}=a_{\mathrm{c}}=1.8 \mathrm{~cm}$ (for high superheating);

$z_{\mathrm{p} 0}=7.5 \mathrm{~cm}$

$\Delta T_{\mathrm{p}}=0-20^{\circ} \mathrm{C}$

$\Omega=0-10 \mathrm{RPM}$

$U_{\mathrm{h}}=0 \mathrm{~cm} / \mathrm{h}$

$U_{\text {amp }}=0.5 \mathrm{~cm} / \mathrm{h}$

$\gamma=0-5^{\circ}$

Dimensionless groups

$\operatorname{Pr}=0.059(\mathrm{GaAs}), 0.02(\mathrm{Ga})$;

$\mathrm{Ra}_{\mathrm{T}}=0-6.9 \times 10^{6} ; \mathrm{Ra}_{\mathrm{w}}=0-7.36 \times 10^{5}$

tion, the major cells compete with each other leading to the periodic oscillation in temperature and zone length. One can also examine the isotherms (or one of the iso-surface) in Fig. 4b to get a better picture of the oscillation. Clearly, similar to the gallium zone, no angular wave is found; the flow structure is also similar to that shown previously in Fig. 2. The growth interface resulted from this flow structure is similar to that due to the one-roll flow (m1 mode). Again, if we apply 10 RPM rotation, we are able to obtain a steady result, as shown in Fig. 5 b. More importantly, the solution is axisymmetric 


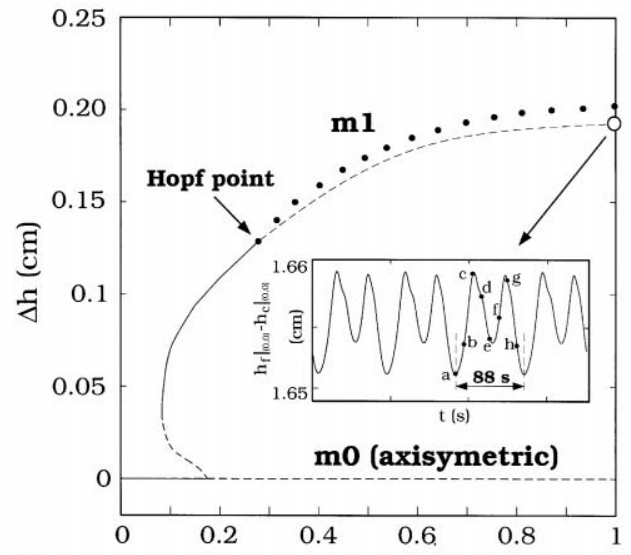

(a) Gravity $\left(\times 980 \mathrm{~cm} / \mathrm{s}^{2}\right)$

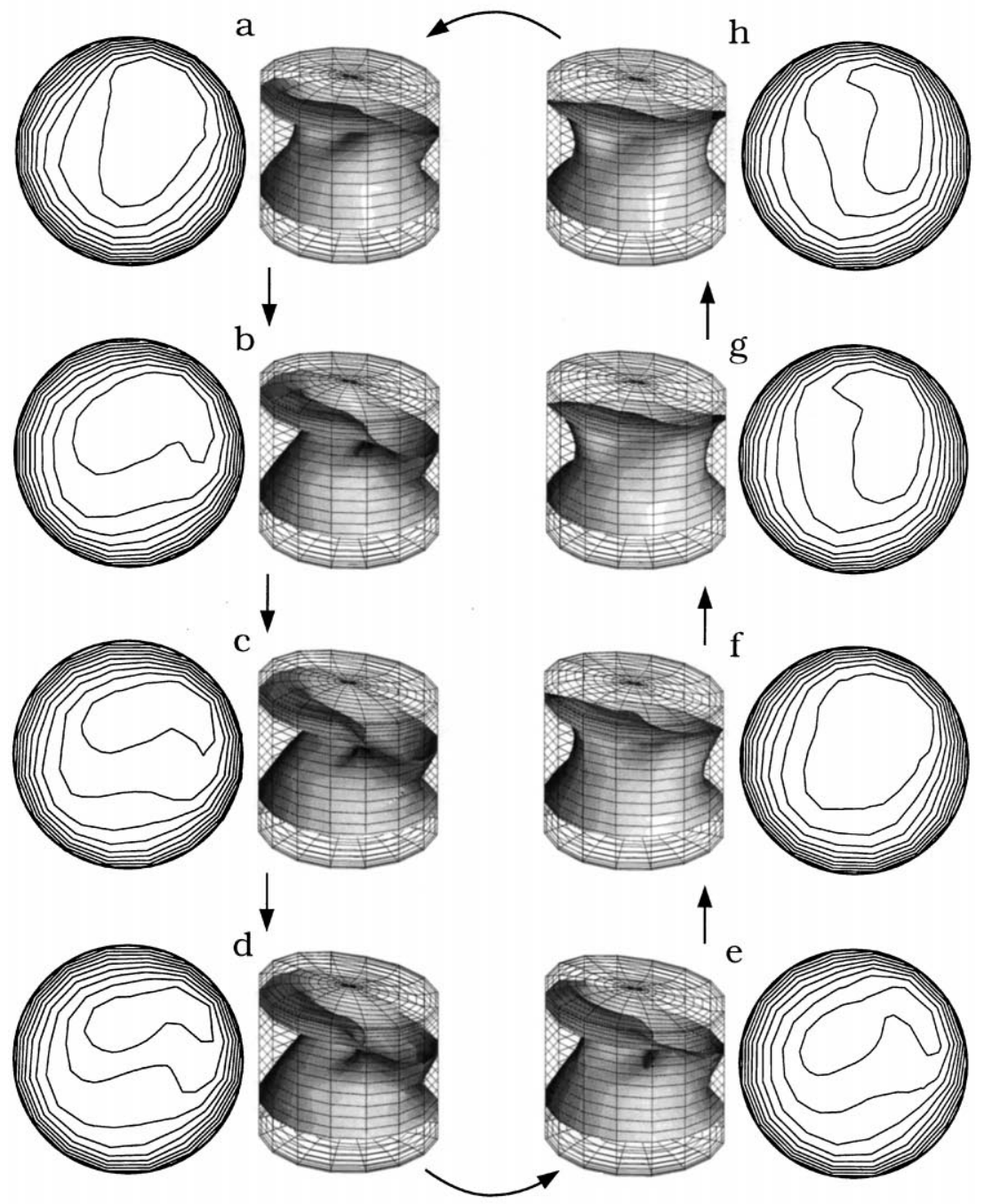

(b) 
(a)

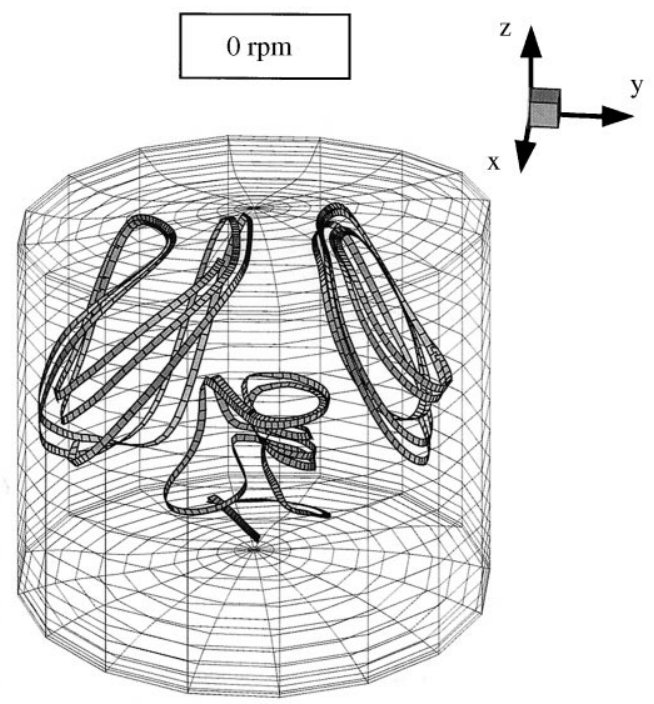

(b)

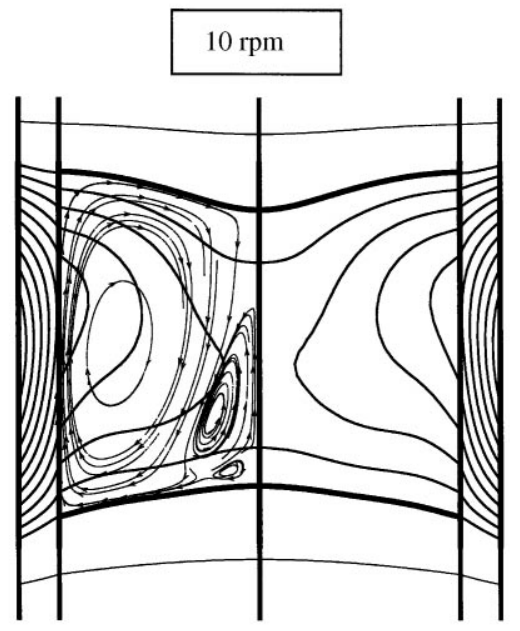

Fig. 5. (a) Typical flow pattern for Fig. 4(b); (b) axisymmetric flow pattern and isotherms after 10 RPM rotation.

(an m0 mode). Again, there is a small cell induced due to the rotation near the center line, and this flow cell seems to hinder other flows from coming across. One can also compare this with the flows in

Fig. 4. (a) Bifurcation diagram of zone length deviation for high superheating and the zone length oscillation at normal gravity $\left(\mathrm{Ra}_{\mathrm{w}}=7.36 \times 10^{5}\right)$; (b) some isotherms showing a period of oscillation. The black dots ( ) in (a) indicate the maximum values of the oscillations. the lower figure of Fig. 2 (10 RPM) to notice the flow cell near the lower center, which is also induced by ampoule rotation. Again, due to this flow structure, the growth front is convex.

In addition to the physical symmetry breaking and unsteadiness, an imperfect growth condition due to ampoule tilting or asymmetric heating may also induce $3 \mathrm{D}$ or unsteady flows. To focus our discussion on the symmetry breaking, a low-superheating configuration $\left(T_{\mathrm{p}}=1500^{\circ} \mathrm{C}\right)$ [9] is taken for consideration here. Under a perfect growth condition $\left(\gamma=0^{\circ}, \Delta T_{\mathrm{p}}=0^{\circ} \mathrm{C}\right)$, a steady axisymmetric solution can be easily obtained, as shown in Fig. 6 a. However, tilting the ampoule slightly can lead to significant 3D flows and melt zone, as shown in Figs. $6 \mathrm{~b}$ and $\mathrm{c}$ for $\gamma=1^{\circ}$ and $5^{\circ}$, respectively. Especially, the flow in Fig. $6 \mathrm{c}$ has a typical $\mathrm{m} 1$ structure. Interestingly, with 10 RPM rotation, as illustrated in Fig. 7, the asymmetry is significantly reduced; the flow structure in Fig. 7c is a toroidal mode (not m1 any more). Also, due to the rotation, the growth interfaces in Fig. 7 are more convex than that in Fig. 6. This is also consistent with the observation by Lan and Chian [15] from their 2D calculations. One can also notice that the rotation pushes the asymmetric isotherms in the angular direction, as shown by the mid-plane isotherms in Fig. 7c. Such an angular distortion of the thermal fields help reduce the asymmetry caused by the tilting. The Stefan effect, i.e., the rotational growth and melting, in the angular direction also has contribution.

Similar improvement is also found for asymmetric heating. Fig. 8 shows the effects of $\Delta T_{\mathrm{p}}\left(0^{\circ}, 5^{\circ}\right.$, and $20^{\circ} \mathrm{C}$, respectively) on the heat flows and the zone shape. As shown in Fig. $8 \mathrm{c}$, only $40^{\circ} \mathrm{C}$ difference in $T_{\mathrm{p}}\left(\Delta T_{\mathrm{p}}=20^{\circ} \mathrm{C}\right)$ can result in a severely distorted molten zone, and the flow structure in also $\mathrm{m} 1$. However, with 10 RPM rotation, as illustrated in Fig. 9, a nearly axisymmetric growth condition can be obtained. One can also examine the isotherms at the mid-plane of the zone. Apparently, the improvement in Fig. 8 seems to be much better than the previous cases. Again, the angular thermal nonuniformity caused by the heater is significantly smoothed out by the rotational effect. Indeed, the thermal difference buildup in the ampoule caused by the nonuniform heating has been significantly reduced by the rotation. One can also examine the 
$\gamma=0^{\circ}$
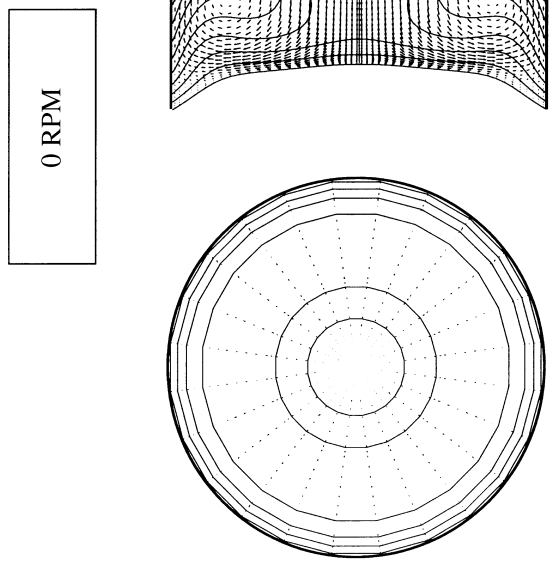

(a) $\gamma=1^{\circ}$
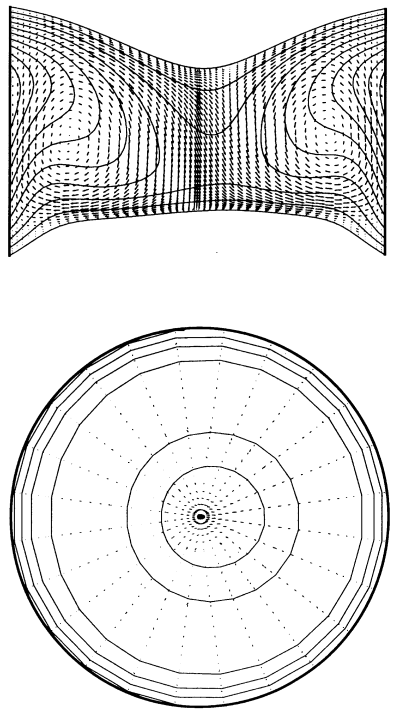

(b) $\gamma=5^{\circ}$
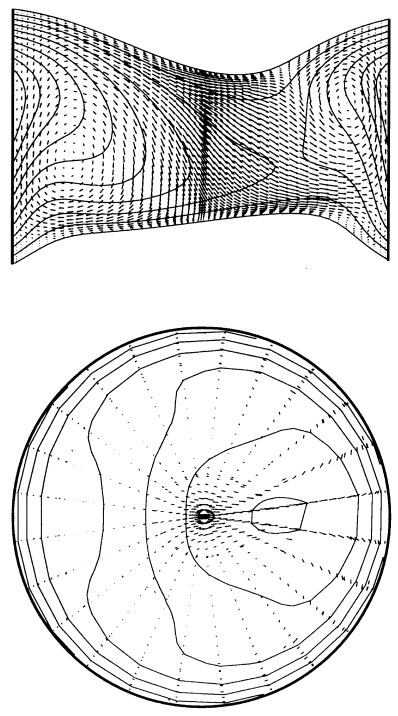

(c)

Fig. 6. Effect of ampoule tilting: (a) $\gamma=0^{\circ}$; (b) $\gamma=1^{\circ}$; (c) $\gamma=5^{\circ}$; $T_{\mathrm{p}}=1500^{\circ} \mathrm{C}$.

$\gamma=0^{\circ}$
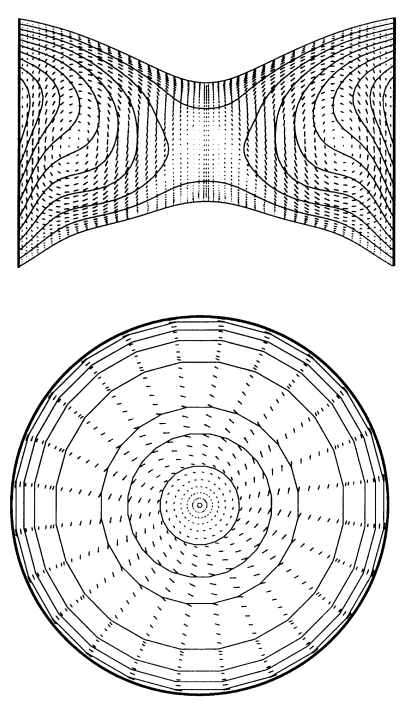

(a) $\gamma=1^{\circ}$
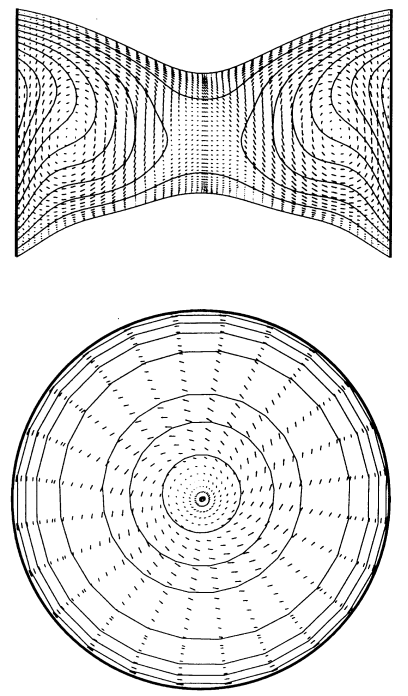

(b)
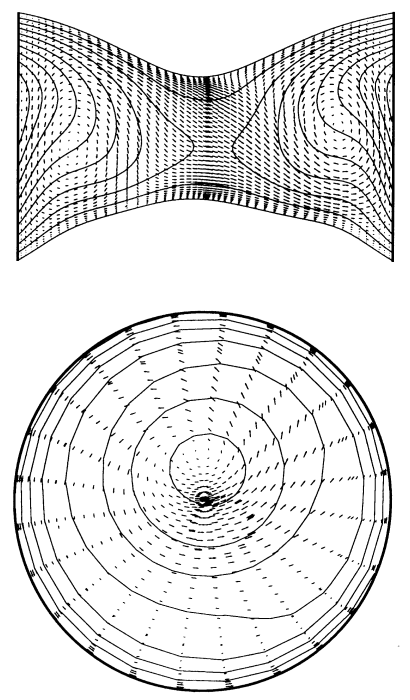

(c)

Fig. 7. Effect of ampoule tilting after $10 \mathrm{RPM}$ rotation: (a) $\gamma=0^{\circ}$; (b) $\gamma=1^{\circ}$; (c) $\gamma=5^{\circ} ; T_{\mathrm{p}}=1500^{\circ} \mathrm{C}$. 
$\Delta \mathrm{T}_{\mathrm{p}}=0^{\circ} \mathrm{C}$

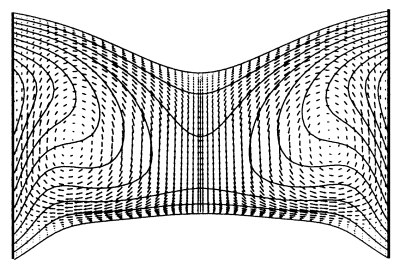

$\sum_{\substack{\alpha \\ 0}}$

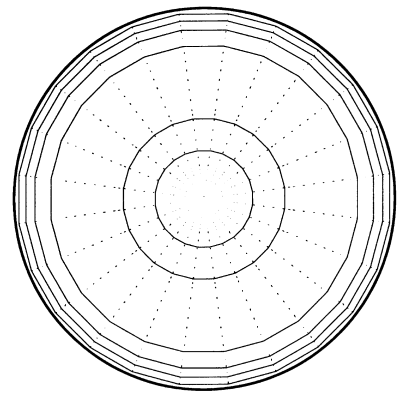

(a)
$\Delta \mathrm{T}_{\mathrm{p}}=5^{\circ} \mathrm{C}$
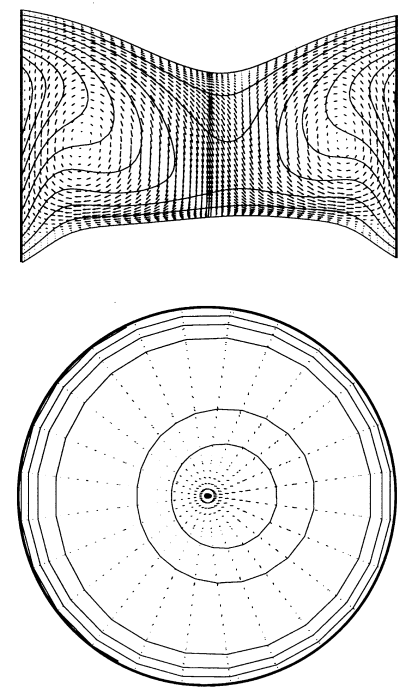

(b)
$\Delta \mathrm{T}_{\mathrm{p}}=20^{\circ} \mathrm{C}$
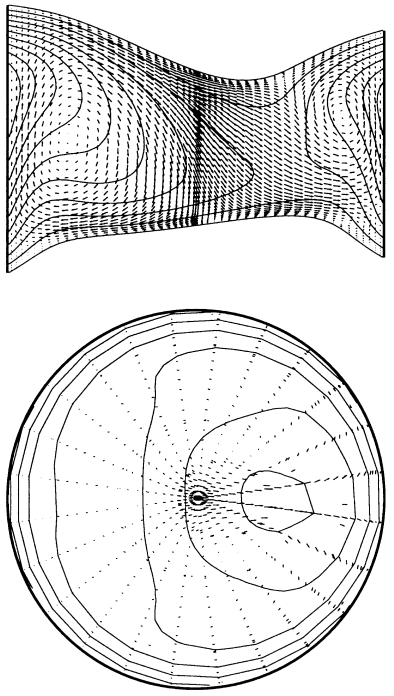

(c)

Fig. 8. Effect of asymmetric heating: (a) $\Delta T_{\mathrm{p}}=0^{\circ}$; (b) $\Delta T_{\mathrm{p}}=5^{\circ}$; (c) $\Delta T_{\mathrm{p}}=20^{\circ} ; T_{\mathrm{p}}=1500^{\circ} \mathrm{C}$.

$$
\Delta \mathrm{T}_{\mathrm{p}}=0^{\circ} \mathrm{C}
$$
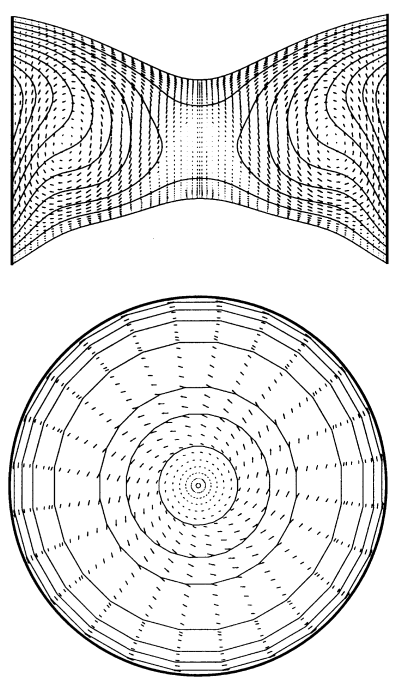

(a)
$\Delta \mathrm{T}_{\mathrm{p}}=5^{\circ} \mathrm{C}$
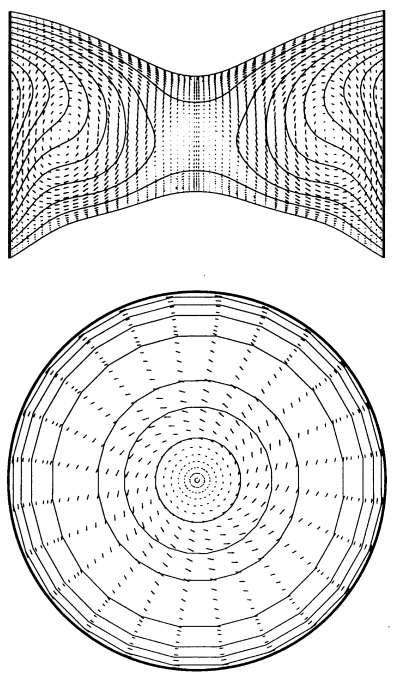

(b)
$\Delta \mathrm{T}_{\mathrm{p}}=20^{\circ} \mathrm{C}$
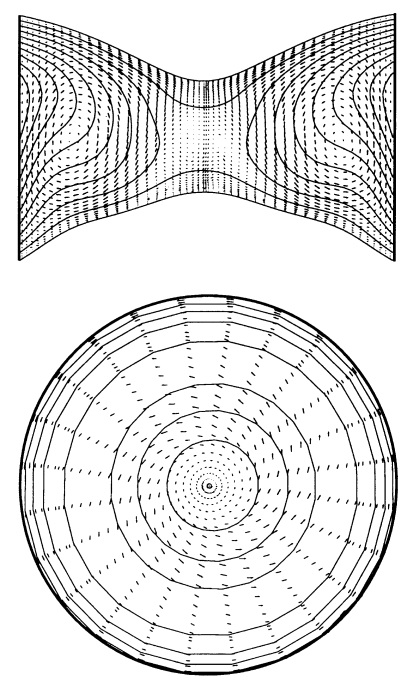

(c)

Fig. 9. Effect of asymmetric heating after $10 \mathrm{RPM}$ rotation: (a) $\Delta T_{\mathrm{p}}=0^{\circ}$; (b) $\Delta T_{\mathrm{p}}=5^{\circ} ;$ (c) $\Delta T_{\mathrm{p}}=20^{\circ} ; T_{\mathrm{p}}=1500^{\circ} \mathrm{C}$.

convective term in Eq. (4) that the angular convective heat transfer enhances the heat transfer in the azimuthal angle direction. As a result, the thermal asymmetry in the ampoule is reduced first. The following angular melt flow further improves the thermal uniformity.

Furthermore, although the ampoule rotation seems to be useful for getting better growth 
conditions for VZM, there are some complications that may arise. Especially, if the co-axis rotation cannot be achieved during growth, the steady-state growth condition cannot be obtained because the zone-to-heater distance is changing with time during rotation. Also, mechanical vibration due to rotation may be a problem as well, and the induced thermovibrational field is not uniform. Furthermore, if the growth interface is not perfectly axisymmetric, continuous remelting and growth proceeds with the rotation. As a result, constitutional supercooling and rotational striations may appear. Therefore, careful implementation of this technique is necessary. An easier approach is to rotate the whole growth system, including the ampoule and the furnace, simultaneously.

\section{Conclusions}

In this study, we have illustrated the significance of steady ampoule rotation, for the first time, on the 3D heat flows during VZM crystal growth. From the calculated results, both for the Ga model zone and the GaAs growth, we find that the 10 RPM ampoule rotation suppresses the 3D characteristics significantly, both on the symmetry breaking and unsteadiness. However, if the unsteadiness is not removed, the introduction of the rotation may cause the baroclinic waves. For an asymmetric growth condition due to ampoule tilting or asymmetric heating, the steady ampoule rotation is also effective in minimizing the asymmetry, especially for the asymmetric heating. Apparently, the angular convective heat transfer reduces the asymmetry and improves the thermal uniformity. Indeed, this is the typical mechanism that is often used in practice to reduce thermal asymmetry by rotation. However, there are other concerns that may appear in practice, especially, for the asymmetric growth interface. Continuous regrowth and remelting, as well as the rotational striations, may be factors that need to be taken into account. Nevertheless, this study provides an alternative that may be worth trying. For high-Prandtl-number materials, the effects of ampoule rotation are still not clear. The baroclinic waves in Czochralski oxide growth $[28,29]$ may occur in VZM due to similar thermal configuration. Furthermore, for an unstable thermal field, the onset of convection under rotation is significantly affected by the Pr value [30]; the Rayleigh-Benard onset is independent of $\mathrm{Pr}$. Therefore, further study for high-Pr materials will be considered in the near future.

In summary, the steady ampoule rotation seems to be an effective way for suppressing 3D unsteadiness during vertical zone melting, at least for $\mathrm{Ga}$ and GaAs. Even though the calculations are carefully performed and the results seem to be reasonable, further experimental verification through a model system or crystal growth experiments is necessary.

\section{Acknowledgements}

The authors are grateful for the support from the National Science Council and the National Center for High Performance Computing of the Republic of China under Grant No. NSC88-2214-E0082006.

\section{References}

[1] W.G. Pfann, Zone Melting, 2nd edition, Wiley, New York, 1966.

[2] E.S. Johnson, J. Crystal Growth 30 (1975) 249.

[3] L. Colombo, R.R. Chang, C.J. Chang, B.A. Baird, Vacuum Sci. Technol. A 6 (1988) 2795.

[4] R.L. Henry, P.E.R. Nordquist, R.J. Gorman, S.B. Qadri, J. Crystal Growth 109 (1991) 228.

[5] C.W. Lan, D.T. Yang, Modeling Simul. Mater. Sci. Eng. 3 (1995) 71.

[6] C.W. Lan, D.T. Yang, Numerical Heat Transfer, Part A 29 (1996) 131.

[7] G. Neumann, J. Fluid Mech. 24 (1990) 59.

[8] J. Baumgartl, W. Budweiser, G. Muller, G. Neumann, J. Crystal Growth 97 (1989) 9.

[9] C.W. Lan, M.C. Liang, J. Crystal Growth 208 (2000) 327.

[10] Q. Xiao, S. Kuppurao, A. Yeckel, J.J. Derby, J. Crystal Growth 167 (1996) 292.

[11] M.C. Liang, C.W. Lan, J. Crystal Growth 167 (1996) 320.

[12] J. Baumgartl, M. Gewald, R. Rupp, J. Stierlen, G. Muller, Proceedings of the Seventh European Symposium on Materials and Fluid Sciences in Microgravity, Oxford, UK, Vol. ESP-295, 1990, p. 47.

[13] M. Yao, A. Chait, A.L. Fripp, W.J. Debnam, J. Crystal Growth 173 (1997) 467.

[14] G. Muller, in: H.C. Freyhardt (Ed.), Crystals, Vol. 12, Springer, Berlin, 1988. 
[15] C.W. Lan, C.H. Chian, J. Crystal Growth 203 (1999) 286.

[16] S. Chandrasekhar, Hydrodynamic and Hydromagnetic Stability, Dover, New York, 1961, p. 76.

[17] Fluent UNS 5.0 Usual Manual, Fluent Inc., Lebanon, New Hampshire, 1999.

[18] C.W. Lan, D.T. Yang, Int. Heat Mass Transfer 41 (1998) 4351.

[19] M.C. Liang, C.W. Lan, J. Comp. Phys. 127 (1996) 330.

[20] C.W. Lan, M.C. Liang, J. Comp. Phys. 152 (1999) 55.

[21] J.H. Ferziger, M. Peric, Computational Methods for Fluid Dynamics, Springer, New York, 1996.

[22] R. Hide, Bull. Am. Meteor. Soc. 47 (1966) 873.

[23] H.P. Greenspan, The Theory of Rotating Fluids, Cambridge University Press, London, 1968.
[24] J.J. Derby, R.A. Brown, J. Crystal Growth 74 (1986) 605.

[25] D.H. Kim, R.A. Brown, J. Crystal Growth 109 (1991) 66.

[26] K. Kakimoto, T. Hibiya, Appl. Phys. Lett. 50 (1987) 1249.

[27] G. Muller, A. Ostrogorsky, in: D.T.J. Hurle (Ed.), Handbook of Crystal Growth 2b: Growth Mechanisms and Dynamics, North-Holland, Amsterdam, 1994, p. 754.

[28] J.C. Brice, T.M. Bruton, O.F. Hill, P.A.C. Whiffin, J. Crystal Growth 24/25 (1974) 429.

[29] Q. Xiao, J.J. Derby, J. Crystal Growth 152 (1995) 169.

[30] H.F. Goldstein, E. Knobloch, I. Mercader, M. Net, J. Fluid Mech. 262 (1994) 293. 